\title{
Using the Wiimote as a sensor in water research
}

\author{
R. W. Hut, ${ }^{1}$ S. V. Weijs, ${ }^{1}$ and W. M. J. Luxemburg ${ }^{1}$ \\ Received 25 March 2010; revised 7 September 2010; accepted 16 September 2010; published 1 December 2010. \\ [1] The $\$ 40$ "Wiimote" (an input device belonging with the Nintendo ${ }^{\circledR}$ Wii ${ }^{\mathrm{TM}}$ game \\ system) can be used by hydrologists as a sensor. The device contains three accelerometers \\ and an infrared camera with built-in source tracking. It communicates by Bluetooth ${ }^{\circledR}$. \\ Because of the efforts of the hacking community it is now easy to let the Wiimote \\ communicate with a standard personal computer. Using a floating evaporation pan as an \\ example, we show that the Wiimote, although it may have potential drawbacks when \\ used in field campaigns, is a good addition to the hydrologist's bag of tools, especially for \\ proof of concept testing.
}

Citation: Hut, R. W., S. V. Weijs, and W. M. J. Luxemburg (2010), Using the Wiimote as a sensor in water research, Water Resour. Res., 46, W12601, doi:10.1029/2010WR009350.

\section{Introduction}

[2] A large collection of sensors is available for hydrological experimentation (see Fraden [2004] for an overview). A limiting factor in employing these sensors is often the number of sensors available in a lab and thus, directly or indirectly, their cost. Scientific sensors are often very expensive, partly due to the inclusion of digital data storage of measurement data or digital interfaces. Because of the high cost of those sensors, they are usually not available for simple proof of concept experiments, or for educational purposes.

[3] With this paper, we wish to draw the attention of the hydrological community to a new low-cost piece of equipment that can be used to conduct measurements: the Nintendo ${ }^{\circledR}$ Wii ${ }^{\mathrm{TM}}$ remote (commonly known as Wiimote). This game console controller contains motion sensors, an infrared camera with position tracking and a wireless connection to a computer by Bluetooth ${ }^{\mathbb{R}}$. In recent years, the (online) hacking community has reverse engineered most of the functionality of this device. The knowledge gained in these efforts has largely been made available online through wikis and Web sites such as http://wiiyourself.gl.tter.org. A large body of software aimed at communicating with the Wiimote is (freely) distributed. Anything presented in this paper benefits greatly from the work of the anonymous individuals who publish on those wikis. The work presented here was sparked by the video (YouTube) tutorials and free software downloads from Johnny Lee, whose work on the Wiimote was recently also published in a scientific journal [Lee, 2008]. Using the software available online (we added logging capabilities to software available at http:// wiiyourself.gl.tter.org/), it is easy to read the observations from the sensors on the Wiimote and store them on a computer. Because of this, the Wiimote offers an opportunity for hydrologists to conduct proof of concept experiments at low cost.

\footnotetext{
${ }^{1}$ Department of Water Management, Faculty of Civil Engineering and Geosciences, Delft University of Technology, Delft, Netherlands.
}

Copyright 2010 by the American Geophysical Union. 0043-1397/10/2010WR009350

\section{Specifications}

[4] The Wiimote is a remarkable game controller in that it contains, along with the regular buttons, a three-axis linear accelerometer and an infrared camera. Lee [2008] gives an overview of the technical specifications of the device. In this section, those relevant for measuring will be repeated.

[5] The accelerometer has a range of $\pm 3 \mathrm{~g}$ and communicates at $100 \mathrm{~Hz}$ in 8 bits per axis. Since gravity is not canceled out in the sensor, this sensor can also be used to retrieve the orientation of the Wiimote relative to the earth gravitational field.

[6] The infrared camera has a resolution of $1024 \times$ 768 pixels and a 45 degree angle of vision (horizontal). The output of the Wiimote is not its recorded image, but the location (measured in pixels) of the 4 brightest infrared sources in its view. Tracking of these infrared sources is done by hardware on the Wiimote.

[7] Communication between the Wiimote and the computer is done by Bluetooth ${ }^{\circledR}$. Connecting a Wiimote to a computer is as straightforward as connecting any other Bluetooth ${ }^{\circledR}$ device. The software mentioned above, although usually designed for a specific purpose, provides easy-to-use interfaces to read out the data that the Wiimote's sensors detect.

\section{Example: Floating Evaporation Pan}

[8] As an example of the capabilities of the Wiimote as a hydrological sensor, we will present some results on water level measurements in a floating evaporation pan. To measure the evaporation from open water bodies, usually an evaporation pan is placed in a contraption that lets it float, while making sure that the water in the pan is at the same temperature as the water in the open water body [Liebe, 2005]. A problem with real-time measurements in this setup is that wave motion (both of the pan on the water and of the water in the pan) prohibits good measurements of the volume of water in the pan with high enough accuracy. To test whether the water level in an evaporation pan can be measured at high sample frequencies, and whether small changes in water level can be retrieved from the 


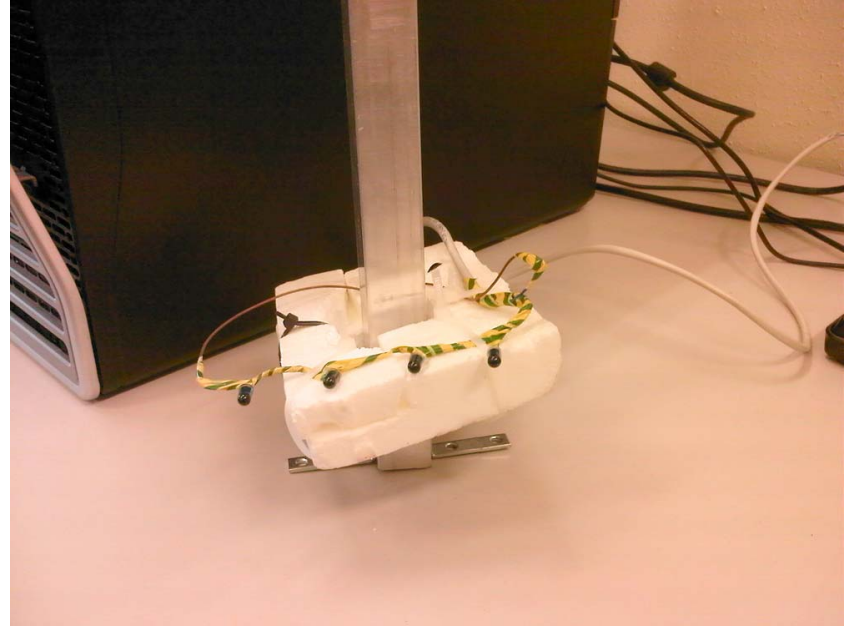

Figure 1. Picture of the float used in the experiment, with the four infrared LEDs clearly visible. The LEDs are bolted to the float with small bolts to make sure they do not move around with respect to the float during heavy wave motion.

recorded signal, we decided to do a proof of concept experiment.

\subsection{Experimental Setup}

[9] The experiment was carried out under controlled circumstances in the wave generator basin at the hydraulics lab of Delft University of Technology. A class-A evaporation pan was strapped to some sheets of styrofoam. In the center of the pan, a steel vertical rod was mounted, along which a little styrofoam float was placed. On the float, 4 infrared LEDs were installed. See Figure 1 for a picture of the float. On the side of the pan, the Wiimote was mounted in a PVC holder, aimed at the float with the LEDs. In this way, the Wiimote "sees" the LEDs and communicates their coordinates with respect to its own field of vision to the laptop. A small hose, connected to a low-flow pump, also fed into the pan. This pump can produce low flows that would result in water level changes of a few millimeters per hour in the pan (mimicking the order of magnitude of the evaporation flux, with opposite sign). See Figure 2 for a schematic overview of the experimental setup.

[10] In the wave generator, waves were generated with an amplitude of about $5-10 \mathrm{~cm}$ and frequencies between 0.5 and $2 \mathrm{~Hz}$. The generator was switched on and off repeatedly to see the effect of increasing and decreasing wave activity. The low-flow pump was set to a flow of $6 \mathrm{~L} / \mathrm{h}$, resulting in a water level rise of $6 \mathrm{~mm} / \mathrm{h}$. The pump was also intermittently activated.

\subsection{Experimental Results}

[11] In this section, selected pieces from the experiment are highlighted to illustrate the opportunities and difficulties when measuring water levels in a floating evaporation pan at high sample frequencies.

[12] In Figure 3 the water levels in the evaporation pan are shown when the wave generator makes $10 \mathrm{~cm}$ (amplitude) waves with a frequency of $1 \mathrm{~Hz}$. In the first part of the experiment the low-flow pump pumps $6 \mathrm{~L} / \mathrm{h}$ into the evaporation pan. The pump and the wave generator are switched off at the same time. The different colors represent the different LEDs. A low-pass, first-order filter with a cutoff frequency of $0.1 \mathrm{~Hz}$ is applied to those signals. The result is shown by the black lines. The output of the first-order filters shows the long-term average, from which the pumping rate of the low-flow pump (simulating evaporation) can be inferred.

[13] In Figure 4 the water levels are shown for a period where the water was initially at rest, and then the wave generator was switched on. Remarkably, the average water level decreases when the wave generator is switched on and it returns to its original level when the generator is switched off. This is attributed to an unknown (nonlinear) effect. Possible explanations include the size of the float compared to the wavelength of the waves in the pan, a nonlinear interference effect, or the (difference in) resistance that the float experiences as it moves along its guiding rod. If a floating evaporation setup was to be part of a field campaign, this effect has to be taken into account. The experiment with the Wiimote showed this in a first test.

\section{Discussion}

[14] In this paper, we have shown that the Wiimote is a useful low-cost addition to the existing set of sensors available to hydrologists, especially for proof of concept testing. Its usefulness is limited by a few shortcomings. First, it needs batteries. A Wiimote can work continuously only for up to around $24 \mathrm{~h}$ on two standard AA batteries. Secondly, it needs a connection to a recording station (usually a regular computer/laptop) with Bluetooth ${ }^{\circledR}$ capabilities. Leaving such a device in the field is not always a viable option and power supply can be a problem. Finally, currently, it is unknown what the temperature and humidity range is in which the Wiimote functions. Because of its design, it functions correctly in ambient indoor environmental ranges, but further work is needed to test its limits. These shortcomings limit the usability of the Wiimote in prolonged field campaigns. In the example of the floating evaporation pan, the Wiimote was used to illustrate that it is possible to record water levels at high frequencies and retrieve the evaporation from a moving pan by filtering. For an extended field experiment, another sensor such as a capacitance probe might be used. The experiment with the Wiimote did show proof of concept in a single day, at very low cost. Furthermore, the

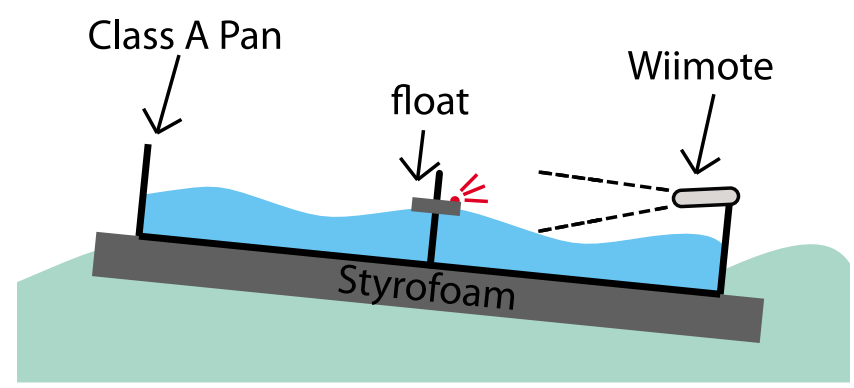

Figure 2. Schematic overview of the experimental setup, showing the class A evaporation pan floating on the waves of the wave generator. The styrofoam float is placed around a rod in the center of the pan. The Wiimote is attached to the side of the pan. The Wiimote "sees" the infrared LEDs on the float and sends their coordinates to a logging laptop using Bluetooth ${ }^{\circledR}$. 


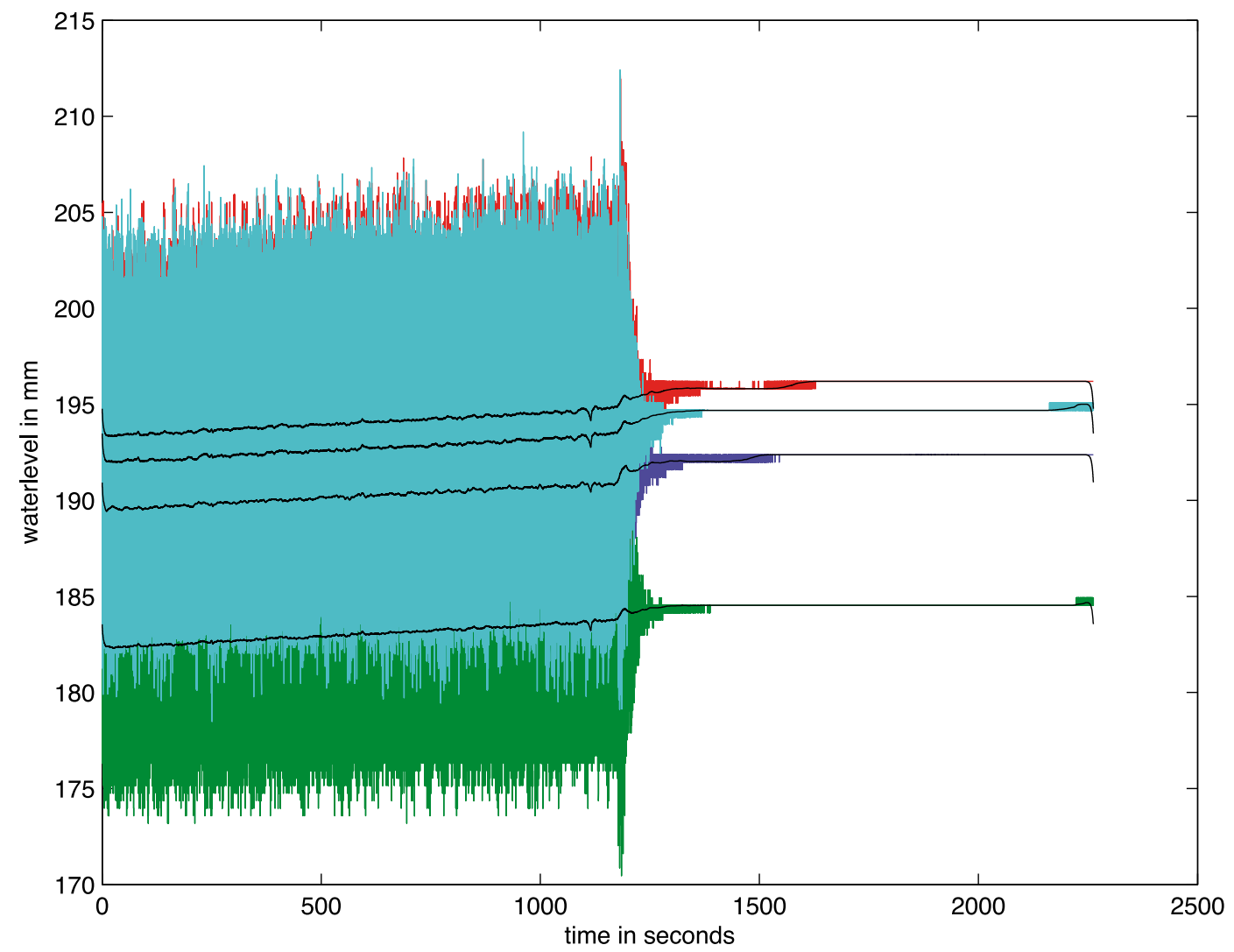

Figure 3. Water level fluctuations in the evaporation pan, as recorded with the Wiimote. In the first part of the measurement both the wave generator and the low-flow pump are active. The wave generator makes waves with a frequency of $1 \mathrm{~Hz}$ and an amplitude of $10 \mathrm{~cm}$. The different colors represent the four different LEDs, and the black lines are the signals, filtered with a first-order low-pass filter with a cutoff frequency of $0.1 \mathrm{~Hz}$. 


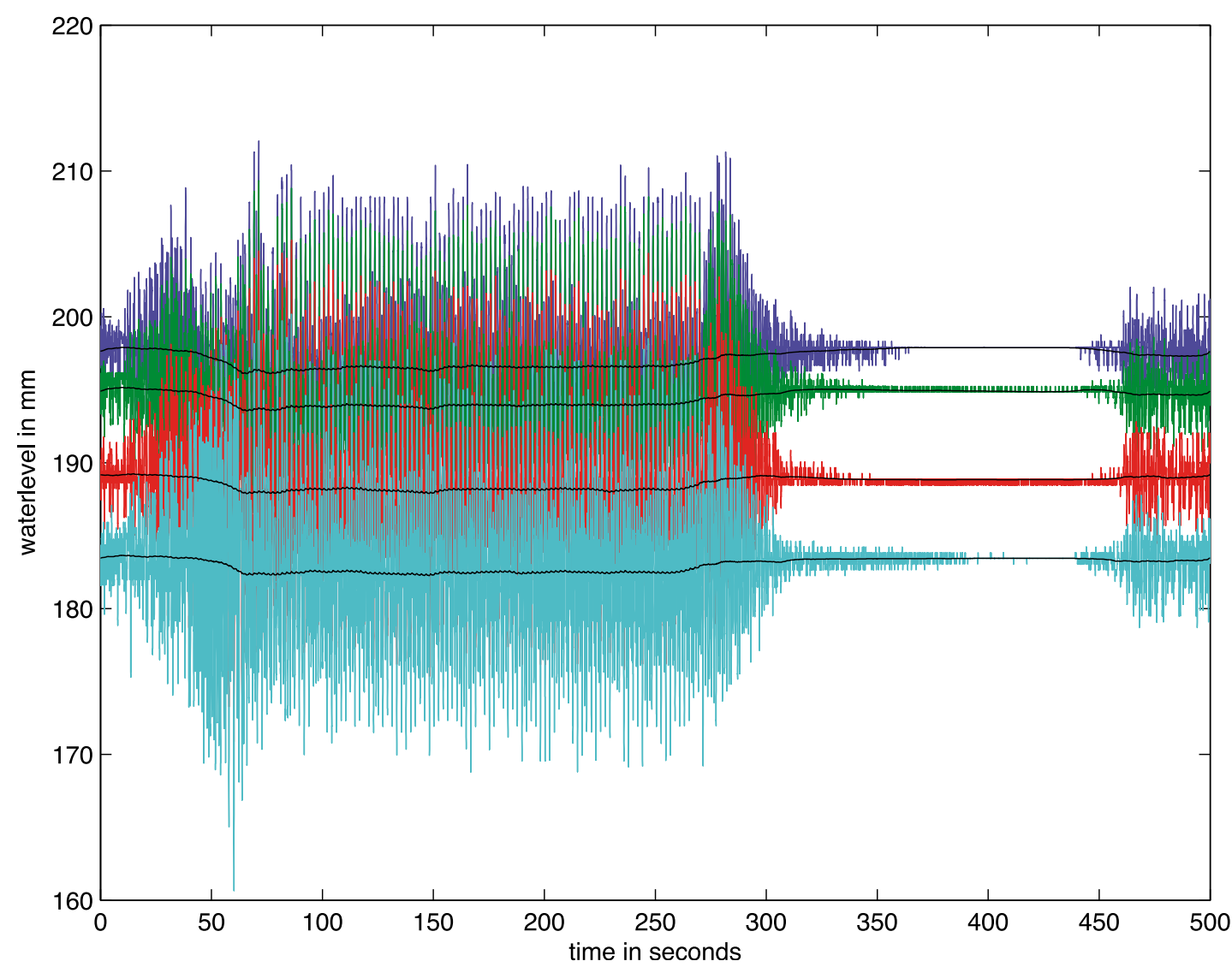

Figure 4. Water level fluctuations in the evaporation pan, as recorded with the Wiimote. In this experiment the low-flow pump was never on and the wave generator was switched on and off. The wave generator makes waves with a frequency of $1 \mathrm{~Hz}$ and an amplitude of $10 \mathrm{~cm}$.

experiment immediately revealed the effect of the timeaveraged water level dropping in the presence of wave movement in the pan. This led to new research questions and more hypothesis to be tested in experiments. All in all, the versatility, low cost and ease of implementation of experiments using the new tool shortened the experimental cycle of experiment-result-conclusions-hypothesis-experiment, which makes it suitable for students' projects and first proofs of concept. Although the Wiimote itself is mainly of interest for such proofs of concept, the technology it uses might have wider use in larger sensor networks. Applications like the one presented in this paper are part of a larger possible shift toward technology transfer from gaming and consumer electronics (e.g., the use of graphic cards for computations). For example, at present many oceanographic sensors have origins in the medical industry, with associated high cost. Technology developed for mass-manufactured consumer electronics, with low cost if acquired in bulk, may offer valuable applications for geosciences, where the spatial density and extent of sensors is often an important constraint on the amount of information that can be obtained from a natural system.

\section{Outlook}

[15] The example experiment presented in section 3 only made use of the infrared motion tracking capabilities of the Wiimote. For future applications in water research, the 3D accelerometers could provide interesting extra data. The floating evaporation pan could for example record the acceleration of the Wiimote to get information about the waves on the lake. Other possible applications could be streamline tracking with infrared reflective floating balls or other motion tracking applications involving up to 4 points in a $2 \mathrm{D}$ plane. Use of multiple Wiimotes opens up the possibilities for inexpensive 3D tracking applications.

[16] Acknowledgments. We would like to thank the reviewers and associate editor who commented on the first version of this paper. Their constructive suggestions helped improve the quality of this paper and pointed out additional insights. Furthermore, we would like to thank technicians at the Waterlab of the Delft University of Technology. Since this paper is on doing experiments at low cost, it might seem counterintuitive to use a large wave generator. However, large measurement setups such as wave generators, once built, are never used full time. The technicians were more than happy to switch the generator on for a while, in between large "official" experiments. If, without this experiment, the wave generator would have been idle, it means that the marginal cost for conducting our simple experiment are very small. We like to encourage this way of looking at costs in research institutes, as opposed to the "pay per minute" attitude normally used for using large experimental equipment, because it acknowledges the economics of sunk costs and compares marginal costs versus marginal benefits.

\section{References}

Fraden, J. (2004), Handbook Of Modern Sensors: Physics, Designs, And Applications, 3rd ed., Springer, New York. 
Lee, J. (2008), Hacking the Nintendo Wii remote, IEEE Pervasive Comput., 7, 39-45.

Liebe, J. (2005), Hydrology of small reservoirs in semi-arid northern Ghana, Ph.D. dissertation, Cornell Univ., Ithaca, N. Y.
R. W. Hut, W. M. J. Luxemburg, and S. V. Weijs, Department of Water Management, Faculty of Civil Engineering and Geosciences, Delft University of Technology, Stevinweg 1, NL-2628 CN Delft, Netherlands. (r.w.hut@tudelft.nl; w.m.j.luxemburg@tudelft.nl; s.v.weijs@ tudelft.nl) 